the histone
H3K27me3
mark
preserves
podocyte
health

$\Rightarrow$ EPIGENETICS

\section{H3K27me3 in glomerular disease}

Epigenetic processes and podocyte dedifferentiation are associated with the development of glomerular disease. New findings now demonstrate that alterations in the histone $\mathrm{H} 3$ lysine 27 trimethylation (H3K27me3) mark in podocytes affects Notch pathway activation and susceptibility to glomerular disease. "We found that the histone H3K27me3 mark preserves podocyte health by preventing reactivation of the developmental Notch pathway," explains Andrew Advani. "In glomerular disease, loss of H3K27me3 causes derepression of the Notch ligand Jagged 1 , which activates the Notch pathway and promotes podocyte dedifferentiation and glomerular injury. Conversely, inhibition of the H3K27me3 demethylating enzymes JMJD3 and UTX causes gain of $\mathrm{H} 3 \mathrm{~K} 27 \mathrm{me} 3$ and attenuates podocyte dedifferentiation."

In settings such as cancer, developmental pathway reactivation and cellular dedifferentiation are regulated by epigenetic mechanisms. This understanding, together with previous work showing that inhibition of the histone $\mathrm{H} 3$ lysine 27 trimethylating enzyme $\mathrm{EZH} 2$ causes podocyte injury in diabetic rats, led Advani and colleagues to examine the role of epigenetic processes in regulating podocyte dedifferentiation in glomerular disease. Using podocyte-specific EZH2-knockout mice and immortalized podocytes together with pharmacological approaches to alter $\mathrm{H} 3 \mathrm{~K} 27 \mathrm{me} 3$ levels, they show that $\mathrm{H} 3 \mathrm{~K} 27 \mathrm{me} 3$ depletion in podocytes derepresses Jagged 1, promoting Notch pathway activation and podocyte dedifferentiation and increasing susceptibility of mice to focal segmental glomerulosclerosis (FSGS) and diabetes-induced kidney injury. By contrast, raising $\mathrm{H} 3 \mathrm{~K} 27 \mathrm{me} 3$ levels attenuated kidney injury in these models. Kidney tissue from patients with diabetic nephropathy or FSGS showed heightened UTX expression, reduced $\mathrm{H} 3 \mathrm{~K} 27 \mathrm{me} 3$ levels and increased Jagged 1 levels. "Our findings extend work on the importance of the Notch pathway in podocyte dedifferentiation by illustrating how this key developmental pathway is regulated by histone patterning in podocytes," says Advani.

Susan J. Allison

ORIGINAL ARTICLE Majumder, S. et al. Shifts in podocyte histone $\mathrm{H} 3 \mathrm{~K} 27 \mathrm{me} 3$ regulate mouse and human glomerular disease. J. Clin. Invest. 128, 483-499 (2018) 